\title{
Study of Hund's rule coupling in models of magnetic impurities and quantum dots
}

\author{
Y Nishikawa ${ }^{1,2}$ and A C Hewson ${ }^{1}$ \\ ${ }^{1}$ Department of Mathematics, Imperial College, London SW7 2AZ, United Kingdom. and \\ ${ }^{2}$ Graduate School of Science, Osaka City University, Osaka 558-8585, Japan
}

\begin{abstract}
Studies of the effects of the Hund's rule coupling $J_{\mathrm{H}}$ in multiple orbit impurities or quantum dots using different models have led to quite different predictions for the Kondo temperature $T_{\mathrm{K}}$ as a function of $J_{\mathrm{H}}$. We show that the differences depend on whether or not the models conserve orbital angular momentum about the impurity site. Using numerical renormalization group (NRG) calculations, we deduce the renormalized parameters for the Fermi liquid regime, and show that, despite the differences between the models, the low energy fixed point in the strong correlation regime is universal with a single energy scale $T_{\mathrm{K}}$, and just two renormalized interaction parameters, a renormalized single orbital term, $\tilde{U}=4 T_{\mathrm{K}}$, and renormalized Hund's rule term, $\tilde{J}_{\mathrm{H}}=8 T_{\mathrm{K}} / 3$.
\end{abstract}

\section{INTRODUCTION}

The role of the Hund's rule coupling $J_{\mathrm{H}}$ has been investigated for magnetic impurities and quantum dots by a number of authors starting from rather different models $1-\underline{\underline{5}}$. This has led to different predictions for the behavior of the Kondo temperature in these systems as a function of $J_{\mathrm{H}}$. As a result it is not clear whether or not the models may also differ in their predictions for their low energy behavior. We investigate this question by applying a combination of the numerical renormalization group (NRG) and renormalized perturbation theory (RPT) to some of these different models. From an analysis of single particle excitations about the NRG low energy fixed point we can obtain an accurate estimate of the Kondo temperature in all cases. We can also calculate the renormalized interaction parameters at this fixed point and compare them for the different models. This information can be used to deduce the low temperature specific heat coefficient, spin and charge susceptibilities and low energy dynamical behavior for the models in all parameter regimes.

A model of a magnetic impurity with $n$-fold degenerate orbitals interaction with a bath of conduction electrons will in general require quite a large number of parameters to describe the interactions between the electrons in these orbitals for various filling factors, and their interaction with the conduction electrons of the host metal. To understand the basic physics of these models a number of different models, using a restricted set of parameters, have been used. One of these models was introduced by Yoshimori (model 1) where the interactions between electrons in the impurity orbitals were specified by just two parameters ${ }^{6}$, a local Coulomb interaction $U$ and an inter-orbital exchange interaction $J_{\mathrm{H}}$. The Hamiltonian of the model takes the form,

$$
\begin{gathered}
\mathcal{H}_{\text {model } 1}=\mathcal{H}_{0}+\mathcal{H}_{d} \\
\mathcal{H}_{0}=\sum_{m \sigma} \epsilon_{d m \sigma} d_{m \sigma}^{\dagger} d_{m \sigma}+\sum_{k, m \sigma} \epsilon_{k m \sigma} c_{k m \sigma}^{\dagger} c_{k m \sigma} \\
+\sum_{k m \sigma}\left(V_{k} d_{m \sigma}^{\dagger} c_{k m \sigma}+V_{k}^{*} c_{k m \sigma}^{\dagger} d_{m \sigma}\right)
\end{gathered}
$$

where $d_{m \sigma}^{\dagger}, d_{m \sigma}$, are creation and annihilation operators for an electron in an impurity state with total an- gular momentum quantum number $l$, and $z$-component $m=-l,-l+1, \cdots, l$, and spin component $\sigma=\uparrow, \downarrow$. The impurity level in a magnetic field $H$ we take as $\epsilon_{d m \sigma}=\epsilon_{d}-\mu_{\mathrm{B}} \sigma H-\mu_{\mathrm{B}} m H-\mu$, where $\sigma=1(\uparrow)$ and $\sigma=-1(\downarrow)$ and $\mu$ is the chemical potential, and $\mu_{\mathrm{B}}$ the Bohr magneton. The creation and annihilation operators $c_{k m \sigma}^{\dagger}, c_{k m \sigma}$ are for partial wave conduction electrons with energy $\epsilon_{k m \sigma}$. The hybridization matrix element for impurity levels with the conduction electron states is $V_{k}$. We denote the hybridization width factor by $\Delta_{m \sigma}(\epsilon)=\pi \sum_{k}\left|V_{k}\right|^{2} \delta\left(\epsilon-\epsilon_{k m \sigma}\right)$, which we can take to be a constant $\Delta$ in the wide flat band limit. The remaining part of the Hamiltonian, $\mathcal{H}_{d}$ describes the interaction between the electrons in the impurity state,

$$
\begin{aligned}
\mathcal{H}_{d}= & \frac{\left(U-J_{\mathrm{H}}\right)}{2} \sum_{m m^{\prime} \sigma \sigma^{\prime}} d_{m \sigma}^{\dagger} d_{m^{\prime} \sigma^{\prime}}^{\dagger} d_{m^{\prime} \sigma^{\prime}} d_{m \sigma} \\
& +\frac{J_{\mathrm{H}}}{2} \sum_{m m^{\prime} \sigma \sigma^{\prime}} d_{m \sigma}^{\dagger} d_{m^{\prime} \sigma^{\prime}}^{\dagger} d_{m \sigma^{\prime}} d_{m^{\prime} \sigma} .
\end{aligned}
$$

This model can be used to describe transition metal impurities, such as Fe or $\mathrm{Mn}$, in a metallic host in the absence of spin orbit or crystal field splittings. The model can be interpreted more generally with $\alpha=m+l+1$ as a channel index taking values $\alpha=1,2, \cdots, n$ where $n=2 l+1$ is the number of channels. The Hund's rule term tends to align the electrons on the impurity site such that for large $U$ and large $J_{\mathrm{H}}$ the impurity state will correspond to a spin $S=n / 2$.

A different $n$-fold model has been studied recently by Nevidomskyy and Coleman ${ }^{2}$ (model 2) which also includes a Hund's rule interaction. This model is characterized by just two interaction terms, an inter-orbital Hund's rule exchange interaction $J_{\mathrm{H}}$ and an Kondo form of exchange interaction $J_{\mathrm{K}}$ between the impurity electrons and the conduction electrons, so that the Hamiltonian takes the form,

$$
\begin{aligned}
H_{\text {model } 2}= & \sum_{k, \alpha \sigma} \epsilon_{k \alpha \sigma} c_{k \alpha \sigma}^{\dagger} c_{k \alpha \sigma}-J_{\mathrm{H}}\left(\sum_{\alpha=1}^{n} \mathbf{s}_{\alpha}\right)^{2} \\
& +J_{\mathrm{K}} \sum_{\alpha=1}^{n} \sum_{k, k^{\prime}} \mathbf{S}_{\alpha} \cdot c_{k \alpha \sigma}^{\dagger} \sigma_{\sigma, \sigma^{\prime}} c_{k^{\prime} \alpha \sigma^{\prime}}
\end{aligned}
$$

There are significant differences between models 1 and 2 , which can be seen more clearly if we restrict the discussion to the case $n=2$, when model 1 can be written in 
a more usual form with the exchange interaction written in terms of spin operators,

$\mathcal{H}_{d, \text { model } 3}=U \sum_{\alpha=1,2} n_{\alpha \uparrow} n_{\alpha \downarrow}+U_{12} \sum_{\sigma \sigma^{\prime}} n_{1 \sigma} n_{2 \sigma^{\prime}}-2 J_{\mathrm{H}} \mathbf{s}_{1} \cdot \mathbf{s}_{2}$.

with $U_{12}=U-3 J_{\mathrm{H}} / 2$. We generalize the model by taking $U_{12}$ as an independent parameter, and refer to the model in this generalized form as model 3. It has been used in this form to describe the interactions in certain double quantum $\operatorname{dots}^{5}$.

We consider, first of all, model 3 in the limit $J_{\mathrm{H}}=0$ with $U_{12}=0$. In this case there is no interaction or hybridization between the two channels and the model is equivalent to two independent Anderson models. In the strong coupling regime $U / \pi \Delta \gg 1$ with particle-hole symmetry it can be converted into model $2\left(J_{\mathrm{H}}=0\right)$ via a Schrieffer-Wolff transformation with $J_{\mathrm{K}}=4 V^{2} / U$. If the first order correction term in $J_{\mathrm{H}}$ is included in this limit, then model 3 can be seen to be essentially equivalent to model 2 in Eq. (4) for $n=2$.

However, for $J_{\mathrm{H}}=0$, and $U_{12}=U$ in Eq. (5), corresponding to the Yoshimori model (model 1), there is an interaction between the two channels. In this case the orbital index $l$ can be combined with the spin index into a single index $(m, \sigma)$ running over 4 values and the model can be shown to have SU(4) symmetry, corresponding to conservation of both spin and orbital angular momentum. In the particle-hole localized limit with $U / \pi \Delta \gg 1$, it can be transformed via a Schrieffer-Wolff transformation (which takes into account virtual excitations from the 2-electron, six-fold degenerate, ground state of the impurity to 1-electron and 3-electron excited states) into an SU(4) Kondo model with $J_{\mathrm{K}}=4 V^{2} / U$ with a six dimensional representation for the local SU(4) operators ${ }^{3}$. Hence models 1 and 2 are quite different in the Kondo regime for $J_{\mathrm{H}}=0$. For general $n$, in the Kondo regime when $J_{\mathrm{H}}=0$, model 1 reduces to an $\mathrm{SU}(2 \mathrm{n})$ Kondo model and model 2 to $n$ independent SU(2) Kondo models. This reflects the important difference that model 1 is invariant under orbital as well as spin rotation, whereas model 2 , and model 3 when $U_{12} \neq U-3 J_{\mathrm{H}} / 2$, are invariant under spin rotation only. This can be verified explicitly by considering the commutator of the generator, $L^{+}=\sum_{\sigma}\left(d_{1, \sigma}^{\dagger} d_{2, \sigma}+c_{k, 1, \sigma}^{\dagger} c_{k, 2, \sigma}\right)$, with the Hamiltonian of model 3. This difference could have consequences for the low energy behavior of these models which we consider in the next section.

\section{LOW ENERGY FERMI LIQUID REGIME}

The conservation of both angular momentum and spin in model 1 permitted Yoshimori ${ }^{6}$ to derive two Ward identities for interaction vertex part at zero frequency. This interaction vertex can be interpretated in terms of a renormalized interaction between the quasiparticles of a Fermi liquid ${ }^{3}$ described by the Hamiltonian,
$\tilde{\mathcal{H}}=\tilde{\mathcal{H}}_{0}+\tilde{\mathcal{H}}_{d}$, where

$$
\begin{aligned}
& \tilde{\mathcal{H}}_{0}=\sum_{m, \sigma} \tilde{\epsilon}_{d, m} \tilde{d}_{m, \sigma}^{\dagger} \tilde{d}_{m, \sigma}+\sum_{k \sigma} \epsilon_{k, m} c_{k, m, \sigma}^{\dagger} c_{k, m, \sigma} \\
& +\sum_{k, m, \sigma}\left(\tilde{V}_{k} \tilde{d}_{m, \sigma}^{\dagger} c_{k, m, \sigma}+\tilde{V}_{k}^{*} c_{k, m, \sigma}^{\dagger} \tilde{d}_{m, \sigma}\right)
\end{aligned}
$$

and

$$
\begin{aligned}
\tilde{\mathcal{H}}_{d}= & \frac{\left(\tilde{U}-\tilde{J}_{\mathrm{H}}\right)}{2} \sum_{m m^{\prime} \sigma \sigma^{\prime}}: \tilde{d}_{m \sigma}^{\dagger} \tilde{d}_{m^{\prime} \sigma^{\prime}}^{\dagger} \tilde{d}_{m^{\prime} \sigma^{\prime}} \tilde{d}_{m \sigma}: \\
& +\frac{\tilde{J}_{\mathrm{H}}}{2} \sum_{m m^{\prime} \sigma \sigma^{\prime}}: \tilde{d}_{m \sigma}^{\dagger} \tilde{d}_{m^{\prime} \sigma^{\prime}}^{\dagger} \tilde{d}_{m \sigma^{\prime}} \tilde{d}_{m^{\prime} \sigma}:
\end{aligned}
$$

This effective model is of the same form as the original model defined in Eqs. (2) and (3) with the difference that the interaction terms have to be normal ordered. The brackets : $\hat{O}$ : indicate the normal ordering of the operator $\hat{O}$ with respect to the ground state of the interacting system, which plays the role of the vacuum. This term only comes into play when more than one quasiparticle is created from the vacuum.

From the Ward identities $3,6,7$ exact expressions can be derived for the impurity charge, spin and orbital susceptibilities, $\chi_{c}, \chi_{s}$, and $\chi_{o r b}$, at $T=0$,

$$
\begin{gathered}
\chi_{c}=2 n \tilde{\rho}^{(0)}(0)\left(1-\left((2 n-1) \tilde{U}-3(n-1) \tilde{J}_{\mathrm{H}}\right) \tilde{\rho}^{(0)}(0)\right) \\
\chi_{s}=2 n \mu_{\mathrm{B}}^{2} \tilde{\rho}^{(0)}(0)\left(1+\left(\tilde{U}+(n-1) \tilde{J}_{\mathrm{H}}\right) \tilde{\rho}^{(0)}(0)\right), \\
\chi_{\text {orb }}=\frac{\left(n^{2}-1\right) \mu_{\mathrm{B}}^{2} \tilde{\rho}^{(0)}(0)}{12}\left(1+\left(\tilde{U}-3 \tilde{J}_{\mathrm{H}}\right) \tilde{\rho}^{(0)}(0)\right),
\end{gathered}
$$

where $\tilde{\rho}^{(0)}(\omega)$ is the free quasiparticle density of states per single spin and channel,

$$
\tilde{\rho}^{(0)}(\omega)=\frac{\tilde{\Delta} / \pi}{\left(\omega-\tilde{\epsilon}_{d, \alpha}\right)^{2}+\tilde{\Delta}^{2}} .
$$

The impurity contribution to the specific heat coefficient $\gamma$ is also given exactly by $\gamma=2 n \pi^{2} \tilde{\rho}^{(0)}(0) / 3$ and the Wilson ratio $R_{\mathrm{W}}=\pi^{2} \chi_{s} / 3 \mu_{\mathrm{B}}^{2} \gamma$ is given by

$$
R_{\mathrm{W}}=1+\left(\tilde{U}+\tilde{J}_{\mathrm{H}}\right) \tilde{\rho}^{(0)}(0) .
$$

In the localized limit $U / \pi \Delta \gg 1$ with particle-hole symmetry $\left(\tilde{\epsilon}_{d}=0\right)$, the charge susceptibility is suppressed so we can equate $\chi_{c}$ to zero. Similarly when $J_{\mathrm{H}}$ is large the orbital susceptibility will be suppressed so that $\chi_{\text {orb }}$ can also be equated to zero. Then from Eqs. (8) and (10) we obtain for the spin susceptibility,

$$
\chi_{s}=\frac{\left(g \mu_{\mathrm{B}}\right)^{2} S(S+1)}{3 T_{\mathrm{K}}}
$$


where $S=n / 2$ and $g=2$, and the relation between the renormalized parameters,

$$
\pi \tilde{\Delta}=\tilde{U}=\frac{3}{2} \tilde{J}_{\mathrm{H}}=4 T_{\mathrm{K}}
$$

As angular momentum is not conserved for model 2, and model 3 if $U_{12} \neq U-3 J_{\mathrm{H}} / 2$, the question arises as to whether the low energy fixed point of this model can be described by a quasiparticle Hamiltonian with renormalized parameters, similar to that given in Eqs. (6) and (77), and if so, does the relation given in Eq. (14) hold when $J_{\mathrm{H}}$ is large?

To examine this question we extend our earlier NRG calculations ${ }^{3}$ of the renormalized parameters for model 1 to models 2 and 3 for the case $n=2$. We can test the hypothesis that the low temperature behaviour of all three models can be described by a quasiparticle Hamiltonian in terms a set of renormalized parameters, $\tilde{\Delta}, \tilde{U}$, $\tilde{U}_{12}$ and $\tilde{J}_{\mathrm{H}}$. We restrict our calculations to the particlehole symmetric case and take $\epsilon_{d}=-U / 2-U_{12}$ in the one-electron part of the Hamiltonian given in Eq. (2). In the NRG calculations we used for the discretization parameter $\Lambda=6$ and half-bandwidth $D=1$, and approximately 4000 states were retained at each iteration. The value $\pi \Delta=0.01$ was used for models 1 and 3 .

\section{CALCULATION OF RENORMALIZED PARAMETERS AND $T=0$ SUSCEPTIBILITIES}

The renormalized parameters that describe the quasiparticles and their interactions can be deduced from an analysis of the low energy NRG fixed point. The parameters $\tilde{\epsilon}_{d}$ and $\tilde{\Delta}$ can be deduced by fitting the lowest single particle and hole excitations from the NRG ground state to those of a non-interacting Anderson model. The interaction parameters, $\tilde{U}, \tilde{U}_{12}$ and $\tilde{J}_{\mathrm{H}}$, can be deduced from the lowest two-particle excitations, in the same channel for $\tilde{U}$, and different channels for $\tilde{U}_{12}$ and $\tilde{J}_{\mathrm{H}}$. We have analysed the fixed point in the same way for all the models, including model 2. Details of these calculations were given in earlier work ${ }^{3,8-10}$ so we shall just quote the results of such an analysis here.

We use the relation, $\pi \tilde{\Delta}=4 T_{\mathrm{K}}$ to deduce the Kondo temperature in the particle-hole symmetric case for all the models. The results for $T_{\mathrm{K}}\left(J_{\mathrm{H}}\right) / T_{\mathrm{K}}(0)$ as a function of $J_{\mathrm{H}} / 4 T_{\mathrm{K}}$ are shown in Fig. 1 for particular parameter sets for the three models. For model 1 with $U / \pi \Delta=5$, the value of $T_{\mathrm{K}}\left(J_{\mathrm{H}}\right)$ falls of rapidly with increase of $J_{\mathrm{H}}$. Results for this case were presented in earlier work ${ }^{3}$, and shown to decrease exponentially with a fit $T_{\mathrm{K}} / \pi \Delta=0.0854 \exp \left(-1.49 \pi^{2} J_{\mathrm{H}} / \pi \Delta\right)$ with $\pi \Delta=0.01$. This exponential dependence is to be expected from a Schrieffer-Wolff transformation in the regime when $U / \pi \Delta \gg 1$ and $J_{\mathrm{H}}$ is large, when the ground state of the isolated impurity is a two electron spin triplet state. When virtual excitations to local states with one more or one less electron are taken into this leads to a spin 1 Kondo model with an effective antiferromagnetic coupling $J_{\mathrm{K}}^{*}=4 V^{2} /\left(U+3 J_{\mathrm{H}}\right)$, giving exponential factor in $J_{\mathrm{H}}$ in the resulting Kondo temperature $T_{\mathrm{K}}^{*} \sim D e^{-1 / 2 J_{\mathrm{K}}^{*} \rho_{c}}$, where $\rho_{c}$ is the density of states of the conduction electrons at the Fermi level.

The results for model 2 are for the case with $J_{\mathrm{K}}=0.3$, and are seen to decrease much more slowly with increase of $J_{\mathrm{H}}$ and appear to level off for larger values of $J_{\mathrm{H}}$. However, the initial values of $T_{\mathrm{K}}(0)$ are rather different for the two models. As model 2 in this limit reduces to $n$-independent $\mathrm{SU}(2)$ Kondo models the Kondo temperature is given by $T_{\mathrm{K}}(0) \sim D e^{-1 / 2 J_{\mathrm{K}} \rho_{c}}$. In this limit, on the other hand, model 1 corresponds to an $\mathrm{SU}(2 \mathrm{n})$ Kondo model, which has a Kondo temperature $T_{\mathrm{K}} \sim D e^{-1 / 2 n J_{\mathrm{K}} \rho_{c}}$, so for the same coupling $T_{\mathrm{K}}(0)$ is likely to be significantly larger than that for the $\mathrm{SU}(2)$ model.

The results for model 3 with $U / \pi \Delta=5, U_{12}=0$, also shown in Fig. 1, are very similar to those of model 2. This is not surprising because because, as noted earlier, when $U_{12}=0$ and $J_{\mathrm{H}}=0$ it can be transformed into model 2 (with $J_{\mathrm{H}}=0$ ) via a Schrieffer-Wolff transformation when $U / \pi \Delta$ is large, and adding the first order correction term in $J_{\mathrm{H}}$ gives essentially model 2 .

Another parameter set for model 3 with $U / \pi \Delta=3$, $U_{12} / \pi \Delta=2.9$, is shown in Fig. 11 In this case there is still a marked fall off of $T_{\mathrm{K}}$ with $J_{\mathrm{H}}$ but intermediate between the other cases shown.

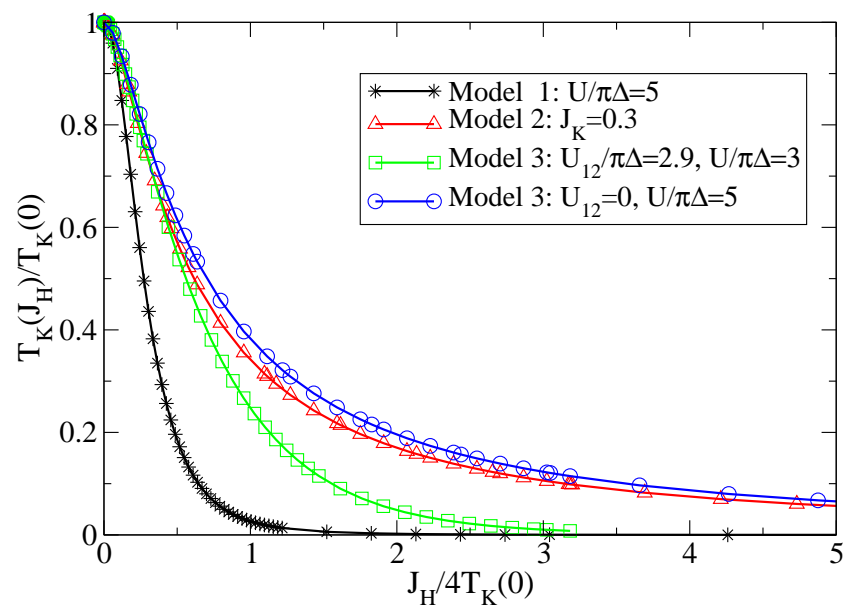

FIG. 1. (Color online) A plot of $T_{\mathrm{K}}\left(J_{\mathrm{H}}\right) / T_{\mathrm{K}}(0)$ versus $J_{\mathrm{H}} / 4 T_{\mathrm{K}}(0)$ for model 1 with $U / \pi \Delta=5$, model 2 with $J_{\mathrm{K}}=0.3$, model 3 with $U_{12} / \pi \Delta=2.9, U / \pi \Delta=3$ and also with $U_{12} / \pi \Delta=0, U / \pi \Delta=5$.

In Fig. 2 we examine the dependence of $T_{\mathrm{K}}$ on $J_{\mathrm{H}}$ for model 2 in more detail by plotting $\ln \left(T_{\mathrm{K}}\left(J_{\mathrm{H}}\right) / T_{\mathrm{K}}(0)\right)$ versus $\ln \left(J_{\mathrm{H}} / 4 T_{\mathrm{K}}(0)\right)$ for a range of values for the Kondo coupling, $J_{\mathrm{K}}=0.1,0.12,0.15,0.2,0.25,0.3$. All the results fall on a universal curve provided $J_{\mathrm{K}}<J_{\mathrm{H}}$. At $J_{\mathrm{K}} \approx J_{\mathrm{H}}$ the curves begin to deviate and develop a 
plateau for $J_{\mathrm{H}}>J_{\mathrm{K}}$ for the larger values of $J_{\mathrm{K}}, J_{\mathrm{K}}=$ $0.15,0.2,0.25,0.3$. Plateaus also develop for the smaller values of $J_{\mathrm{K}}$ for $J_{\mathrm{H}} / T_{\mathrm{K}}(0)$ beyond the range shown in Fig. 2. We can compare these results with the scaling relation derived to one loop order for this model by Nevidomskyy and Coleman ${ }^{2}$ (Eq. (11) in their paper with $n=2$ ) for intermediate values of the Hund's rule coupling $J_{\mathrm{H}}$, which predicts $T_{\mathrm{K}}\left(J_{\mathrm{H}}\right) \propto T_{\mathrm{K}}^{2}(0) / J_{\mathrm{H}}$. This would correspond to a linear behavior in the log-log plot in Fig. 2 with a slope -1 . The dashed line Fig. 2 corresponding to $T_{\mathrm{K}}\left(J_{\mathrm{H}}\right)=4 T_{\mathrm{K}}^{2}(0) / e^{2} J_{\mathrm{H}}$ does fit the curves over a substantial range, particularly for the smaller values of $J_{\mathrm{K}}$. However, a linear fit to the range $J_{\mathrm{H}}<J_{\mathrm{K}}$ for larger values of $J_{\mathrm{K}}$ would give a larger slope with $T_{\mathrm{K}}\left(J_{\mathrm{H}}\right) \propto\left(J_{\mathrm{H}}\right)^{-x}$, with $x$ varying $1<x<1.25$ depending on the fitting range chosen. The linear fit with $x \approx 1$ begins at values of $J_{\mathrm{H}} \sim 4 T_{\mathrm{K}}(0)$.

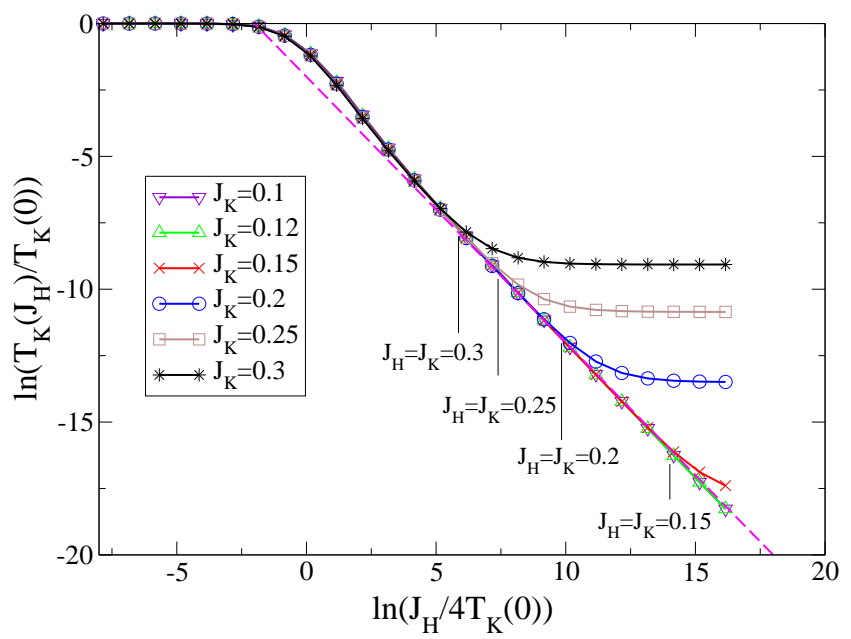

FIG. 2. (Color online) The graph shows a plot of $\ln \left(T_{\mathrm{K}}\left(J_{\mathrm{H}}\right) / T_{\mathrm{K}}(0)\right)$ versus $\ln \left(J_{\mathrm{H}} / 4 T_{\mathrm{K}}(0)\right)$ for model 2 for values of $J_{\mathrm{K}}=0.1,0.12,0.15,0.2,0.25,0.3$. The dashed line corresponds to $T_{\mathrm{K}}\left(J_{\mathrm{H}}\right)=4 T_{\mathrm{K}}^{2}(0) / e^{2} J_{\mathrm{H}}$, on using the relation $4 T_{\mathrm{K}}\left(J_{\mathrm{H}}\right)=\pi \tilde{\Delta}\left(J_{\mathrm{H}}\right)$. The deviation from the approximate linear dependence to a plateau region for $J_{\mathrm{K}}=0.15,0.2,0.25,0.3$ occurs when $J_{\mathrm{H}} \approx J_{\mathrm{K}}$ in all four cases.

When $J_{\mathrm{H}} \gg J_{\mathrm{K}}$ and the two impurity spins are locked together, model 2 should also be equivalent to an effective Kondo model with the electrons in the two channels coupled to a spin $S=1$. For the general $n$-channel model this Hamiltonian would take the form,

$$
H=J_{\mathrm{K}}^{*} \sum_{m=1}^{n} \sum_{k, k^{\prime}} \mathbf{S} . c_{k m \sigma}^{\dagger} \sigma_{\sigma, \sigma^{\prime}} c_{k^{\prime} m \sigma^{\prime}}
$$

with an effective coupling $J_{\mathrm{K}}^{*}$ of a spin $S=n / 2$ to the conduction electrons. In the limit $J_{\mathrm{H}} \rightarrow \infty$ the result, $J_{\mathrm{K}}^{*}=J_{\mathrm{K}} / n$, derived by Schrieffer ${ }^{1}$ for model 2 should be valid. The Kondo temperature of the model with $n=2 S$ would then be given by $T_{\mathrm{K}}^{*} \sim D e^{-1 / 2 J_{\mathrm{K}}^{*} \rho_{c}}$, neglecting any dependence of the prefactors on $J_{\mathrm{K}}^{*}$. The corresponding result for the model with $J_{\mathrm{H}}=0, T_{\mathrm{K}}(0) \sim D e^{-1 / 2 J_{\mathrm{K}}} \rho_{c}$, again neglecting any dependence of the prefactors on $J_{\mathrm{K}}$, would then imply $T_{\mathrm{K}}^{*} \propto\left(T_{\mathrm{K}}(0)\right)^{n}$. We can test this result for $n=2$ by modifying the plot given in Fig. 2 and plot instead $\ln \left(T_{\mathrm{K}}\left(J_{\mathrm{H}}\right) / T_{\mathrm{K}}^{2}(0)\right)$ versus $\ln \left(J_{\mathrm{H}} / 4 T_{\mathrm{K}}(0)\right)$. The results are shown in Fig. 3 for $J_{\mathrm{K}}=0.3,0.25,0.2$, It can be seen for the cases shown that, in the regime $J_{\mathrm{H}}>J_{\mathrm{K}}$, the ratios of $T_{\mathrm{K}}\left(J_{\mathrm{H}}\right) / T_{\mathrm{K}}^{2}(0)$ become independent of the value of $J_{\mathrm{K}}$ which implies that, for $J_{\mathrm{H}} \gg J_{\mathrm{K}}, T_{\mathrm{K}}^{*} \propto\left(T_{\mathrm{K}}(0)\right)^{2}$. This gives a clear criterion $J_{\mathrm{H}}>J_{\mathrm{K}}$ for the Schrieffer relation ${ }^{1}, J_{\mathrm{K}}^{*}=J_{\mathrm{K}} / n$, to hold.

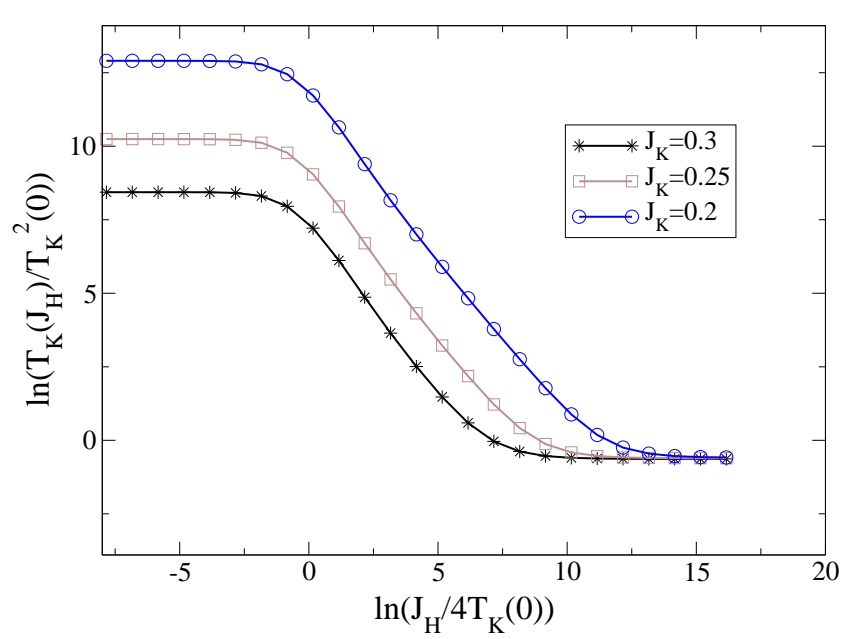

FIG. 3. (Color online) A plot of $\ln \left(T_{\mathrm{K}}\left(J_{\mathrm{H}}\right) / T_{\mathrm{K}}^{2}(0)\right)$ versus $\ln \left(J_{\mathrm{H}} / 4 T_{\mathrm{K}}(0)\right)$ for model 2 for values of $J_{\mathrm{K}}=0.3,0.25,0.2$. The results indicate that the ratio $T_{\mathrm{K}}\left(J_{\mathrm{H}}\right) / T_{\mathrm{K}}^{2}(0)$ becomes independent of $J_{\mathrm{K}}$ in the regime $J_{\mathrm{H}}>J_{\mathrm{K}}$.

In Fig. 4 we plot the ratio of the renormalized parameters, $\widetilde{J}_{H}\left(J_{\mathrm{H}}\right) / \pi \tilde{\Delta}\left(J_{\mathrm{H}}\right)$ versus $J_{H} / 4 T_{\mathrm{k}}(0)$ for the different models for the parameter sets given in Fig. 1. We can see that all the curves asyptotically approach the value predicted in Eq. (14), $\tilde{J}_{H}\left(J_{\mathrm{H}}\right) / \pi \tilde{\Delta}\left(J_{\mathrm{H}}\right) \rightarrow 2 / 3$ on increasing $J_{\mathrm{H}}$. Though they approach this value at different rates they are all very close to the limiting value for $J_{\mathrm{H}}>5 T_{\mathrm{K}}(0)$. This clearly shows that the crossover to $T=0$ susceptibility corresponding to a spin 1 Kondo model, as given in Eq. (13), on increasing $J_{\mathrm{H}}$ occurs on a scale $T_{\mathrm{K}}(0)$. The regime $5 T_{\mathrm{K}}<J_{\mathrm{H}}<J_{\mathrm{K}}$ for model 2 corresponds to the scaling regime $T_{\mathrm{K}}\left(J_{\mathrm{H}}\right) \propto 1 / J_{\mathrm{H}}$ of Nevidomskyy and Coleman ${ }^{2}$ where the low temperature behaviour can be described by a spin 1 Kondo model before the crossover to the regime $J_{\mathrm{H}}>J_{\mathrm{K}}$ where $T_{\mathrm{K}}\left(J_{\mathrm{H}}\right)$ becomes independent of $J_{\mathrm{H}}$, and the Schrieffer result ${ }^{1}$ $J_{\mathrm{K}}^{*}=J_{\mathrm{K}} / 2, T_{\mathrm{K}}^{*} \propto T_{\mathrm{K}}^{2}(0)$, holds.

The remaining renormalized parameter ratios, $\tilde{U} / \pi \tilde{\Delta}\left(J_{\mathrm{H}}\right)$ and $\tilde{U}_{12} / \pi \tilde{\Delta}\left(J_{\mathrm{H}}\right)$, that characterize the low energy fixed point, are shown in Fig. 5 for the different models as a function of $J_{\mathrm{H}} / 4 T_{\mathrm{K}}(0)$. For model 1 with 
$U / \pi \Delta=5$ the charge fluctuations are suppressed $\chi_{c} \sim 0$, so from Eq. (8) when $J_{\mathrm{H}}=0\left(\tilde{J}_{\mathrm{H}}=0\right)$ for $n=2$ we get $\tilde{U} / \pi \tilde{\Delta}=1 / 3$. As $U_{12}=U-3 J_{\mathrm{H}} / 2$ for this model when $J_{\mathrm{H}}=0$, we also predict the result $\tilde{U}_{12} / \pi \tilde{\Delta}=1 / 3$. These are confirmed in the results given in Fig. 5. When $J_{\mathrm{H}}$ is increased and the orbital fluctuations are also suppressed we get the results for this model given in Eq. (14), which imply that in this limit $U_{12} \rightarrow 0$, and the NRG calculations confirm these results.

The NRG results for model 2 give $\tilde{U} / \pi \tilde{\Delta}=1$ and $U_{12}=0$ for all values of $J_{\mathrm{H}}$. We also obtain the same results for model 3 (not shown) for the case $U_{12}=0$, $U / \pi \Delta=5$. This is not surprising because, as noted earlier, when $U_{12} \rightarrow 0$ and $U_{12} / \pi \Delta \gg 1$, model 3 and model 2 are very similar.

Finally in Fig. 5 results are shown for model 3 for the case $U_{12} / \pi \Delta=2.9$ and $U / \pi \Delta=3$. To test the predictions in this case, the expression for $\chi_{c}$ given in Eq. (8) must be generalized as it assumes the relation. $U_{12}=U-3 J_{\mathrm{H}} / 2$. As both charge and spin are conserved in model 3, from the corresponding Ward indentities, exact results for the charge and spin susceptibilities can be derived. For $n=2$ the generalized expression for the charge susceptibility takes the form,

$$
\left.\chi_{c}=4 \tilde{\rho}^{(0)}(0)\left(1-\tilde{U}-2 \tilde{U}_{12}\right) \tilde{\rho}^{(0)}(0)\right) .
$$

As the charge fluctations are suppressed for model 3 with $U_{12} / \pi \Delta=2.9$ and $U / \pi \Delta=3$, Eq. (16) predicts the result $\tilde{U} / \pi \tilde{\Delta}+2 \tilde{U}_{12} / \pi \tilde{\Delta}=1$, which is satisfied in the results shown in Fig. [5] for all values of $J_{\mathrm{H}}$. Though both $\tilde{U} / \pi \tilde{\Delta}$ and $\tilde{U}_{12} / \pi \tilde{\Delta}$ are non-zero for $J_{\mathrm{H}}=0$, as $J_{\mathrm{H}}$ is increased $\tilde{U} / \pi \tilde{\Delta} \rightarrow 1, \tilde{U}_{12} / \pi \tilde{\Delta} \rightarrow 0$ in line with the result in Eq. (14).

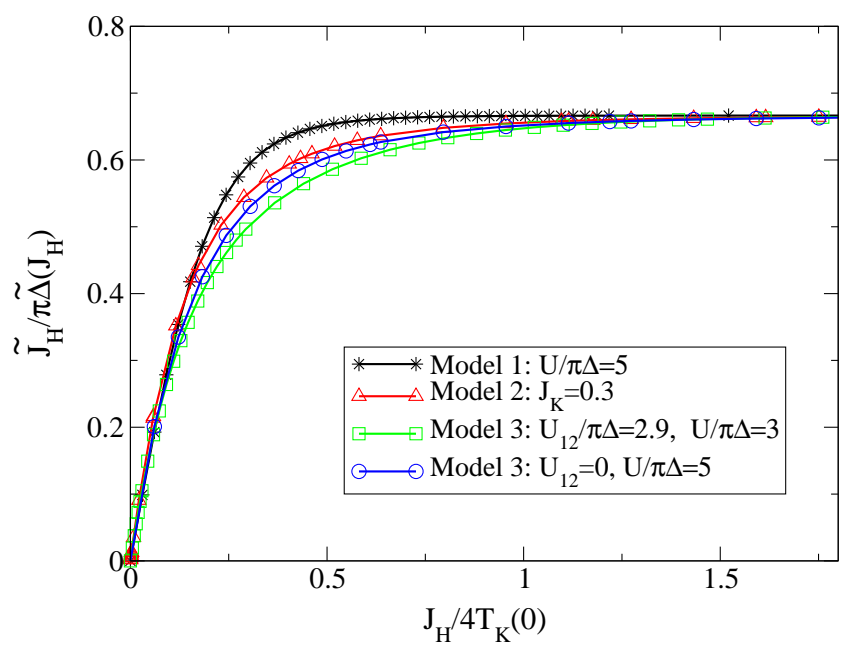

FIG. 4. (Color online) A plot of $\tilde{J}_{\mathrm{H}} / \pi \tilde{\Delta}\left(J_{\mathrm{H}}\right)$ versus $J_{\mathrm{H}} / 4 T_{\mathrm{K}}(0)$ for the models and parameter sets given in Fig. 1]

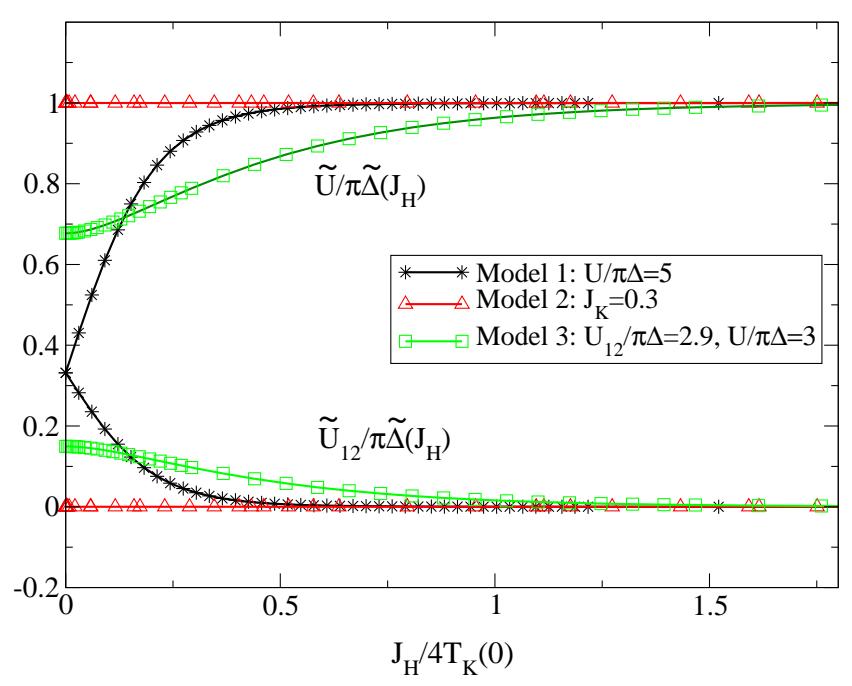

FIG. 5. (Color online) A plot of $\tilde{U} / \pi \tilde{\Delta}\left(J_{\mathrm{H}}\right)$ and $\tilde{U}_{12} / \pi \tilde{\Delta}\left(J_{\mathrm{H}}\right)$ versus $J_{\mathrm{H}} / 4 T_{\mathrm{K}}(0)$ for the models and first three data sets shown in Fig. 1. The corresponding results for the last data set given in Fig. 1 is $\tilde{U} / \pi \tilde{\Delta}\left(J_{\mathrm{H}}\right)=1$ and $\tilde{U}_{12} / \pi \tilde{\Delta}\left(J_{\mathrm{H}}\right)=0$ for all values of $J_{\mathrm{H}}$ in the range shown.

The expressions for spin susceptibility $\chi_{s}$ and Wilson ratio $R_{\mathrm{W}}$ for model 3 with $U_{12} \neq U-3 J_{\mathrm{H}} / 2$ remain unchanged from those given in Eqs. (9) and (12). To calculate the impurity spin susceptibility for the models and parameters sets used in the other plots we substitute the renormalized parameters into Eq. (9) and give the results in Fig. 6. The exponential decrease of the Kondo temperature with increase of $J_{\mathrm{H}}$ gives a dramatic rise in $\chi_{s}$ for model 1 compared with the corresponding results for models 2 and 3 . However, the change in the corresponding Wilson ratios on increasing $J_{\mathrm{H}}$ from zero is much less dramatic. For model 1 with $U / \pi \Delta=5, R_{\mathrm{W}}$ increases from $4 / 3$ for $J_{\mathrm{H}}=0$ to $8 / 3$ for large $J_{\mathrm{H}}$, and for model 2 from 2 to $8 / 3$ over the same range.

Yoshimori ${ }^{6}$ derived an exact result for the low temperature impurity contribution to the resistivity for model 1 in the particle-hole symmetric case and $H=0$. In terms of the renormalized parameters, the result is

$$
R(T)=R_{0}\left(1-\frac{\pi^{4}(1+I)}{48}\left(\frac{T}{T_{\mathrm{K}}}\right)^{2}+\mathrm{O}\left(T^{4}\right)\right)
$$

where $I$ is given by

$$
I=2\left((2 n-1) \tilde{U}^{2}-6(n-1) \tilde{J}_{\mathrm{H}}\left(\tilde{U}-\tilde{J}_{\mathrm{H}}\right)\right) /(\pi \tilde{\Delta})^{2},
$$

and $\pi \tilde{\Delta}=4 T_{\mathrm{K}}$. For models 2 and 3 for the case $n=2$ this result can be generalized using renormalized perturbation 


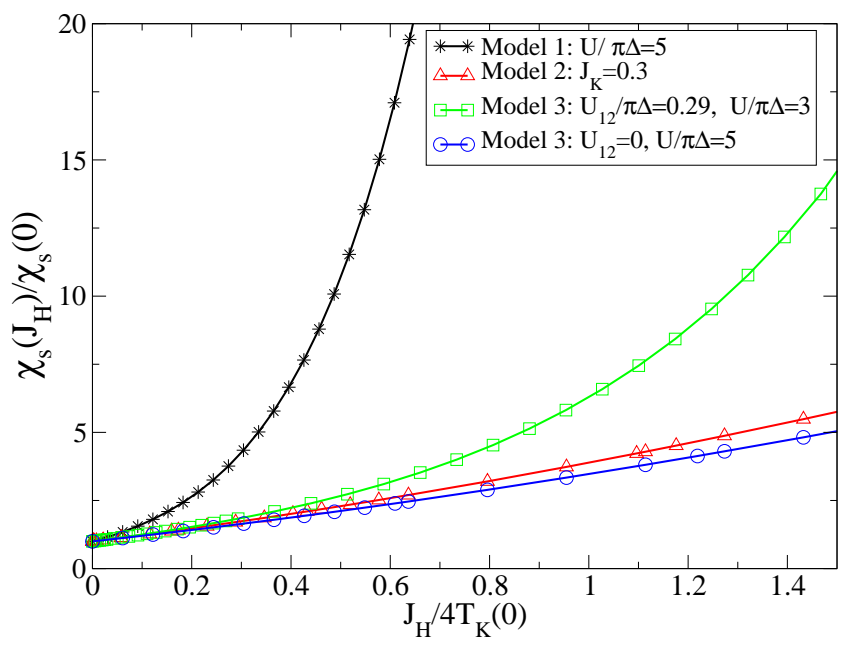

FIG. 6. (Color online) A plot of $\chi_{s}\left(J_{\mathrm{H}}\right) / \chi_{s}(0)$ versus $J_{\mathrm{H}} / 4 T_{\mathrm{K}}(0)$ for the models for the data sets shown in Fig. 1

to second order in the renormalized interaction vertices for the impurity self-energy ${ }^{3,10,11}$ to give

$$
I=\left(2 \tilde{U}^{2}+4 \tilde{U}_{12}^{2}+3 \tilde{J}_{\mathrm{H}}^{2}\right) /(\pi \tilde{\Delta})^{2},
$$

which takes the value $I=10 / 3$ in the regime where Eq. (14) holds.

\section{CONCLUSIONS}

We have shown that we can get very different results for the Kondo temperature as a function of the Hund's rule coupling $J_{\mathrm{H}}$ depending on how this term is included in a model of a magnetic impurity. The physically most relevant model for a magnetic impurity with $n$-fold degenerate states in the absence of crystal field or spin-orbit splitting should be the one introduced by Yoshimori 6 which conserves orbital angular momentum about the impurity site, or the generalizations of this model considered by Mihály and Zawadowsky ${ }^{12}$, and Yoshimori and Zawadowski ${ }^{7}$, where off-diagonal matrix elements of the scattering between orbital channels are included, subject to the condition of overall conservation of angular momentum. These matrix elements omitted from the Yoshimori model have been shown by Noziéres and Blandin 13 not to affect the results in low temperature Fermi liquid regime, nor the derivation of the impurity spin and orbital susceptibilities based on the Ward identities deduced from conservation of spin and orbital angular momentum ${ }^{7}$. The restriction to models where angular momentum is conserved about the impurity site might not be appropriate to all situations, such as use of the model 3 (given in Eq. (44)) to describe the interaction between two quantum $\operatorname{dots}^{\frac{5}{5}}$. We have studied in particular the model used by Nevidomskyy and Coleman ${ }^{2}$ for the case $n=2$ and, for the smaller values of $J_{\mathrm{K}}$, verified their scaling equation $T_{\mathrm{K}} \propto 1 / J_{\mathrm{H}}$ when the Hund's rule coupling becomes strong enough to lock the individual spins to form an effective spin 1 coupled to the conduction electrons with an effective Kondo coupling $J_{\mathrm{K}}^{*}$. We also found the point $J_{\mathrm{H}} \sim J_{\mathrm{K}}$ where this scaling crosses over to the regime considered by Schrieffer ${ }^{1}$, where $J_{\mathrm{K}}^{*}=J_{\mathrm{K}} / n$ and the $T_{\mathrm{K}}$ becomes independent of $J_{\mathrm{H}}$.

We find that the low temperature behaviour of all the models considered can be described in terms of a quasiparticle Hamiltonian with renormalized parameters, which, for the case $n=2$, can be deduced from an analysis of the low energy fixed point of the NRG calculation. We have shown that, despite the differences between the models, in the strong Hund's rule regime, just two interaction parameters are required, a renormalized interaction $\tilde{U}$, within each impurity orbital, and a renormalized Hund's rule interaction between electrons in different orbitals $\tilde{J}_{\mathrm{H}}$, which can both be expressed in terms of the Kondo temperature, $\tilde{U}=4 T_{\mathrm{K}}$ and $\tilde{J}_{\mathrm{H}}=8 T_{\mathrm{K}} / 3$.

\section{Acknowledgment}

We thank Daniel Crow and Johannes Bauer for helpful discussions. This work has been supported in part by the EPSRC Mathematics Platform grant EP/1019111/1.
1 J. R. Schrieffer, Journal of Applied Physics 38, 1143 (1967).

2 A. H. Nevidomskyy and P. Coleman, Phys. Rev. Lett. 103, 147205 (2009).

3 Y. Nishikawa, D. J. G. Crow, and A. C. Hewson, Phys. Rev. B 82, 115123 (2010).

4 T. Pruschke and R. Bulla, Eur. Phys. J. B 44, 217 (2005).

${ }^{5}$ R. Sakano, Y. Nishikawa, A. Oguri, A. C. Hewson, and S. Taruchi, Phys. Rev. Lett. 108, 266401 (2012).

6 A. Yoshimori, Prog. Theor. Phys. 55, 66 (1976).

7 A. Yoshimori and A. Zawadowski, J. Phys. C 15, 5421 (1982).
8 A. C. Hewson, A. Oguri, and D. Meyer, Eur. Phys. J. B 40, 177 (2004).

9 Y. Nishikawa, D. J. G. Crow, and A. C. Hewson, Phys. Rev. B 82, 245109 (2010).

10 Y. Nishikawa, D. J. G. Crow, and A. C. Hewson, Phys. Rev. B 86, 123134 (2012).

11 Y. Nishikawa, D. J. G. Crow, and A. C. Hewson, Phys. Rev. Lett. 108, 056402 (2012).

12 L. Mihály and A. Zawadowsky, J. Physique Lett. 39, L483 (1978).

13 P. Nozières and A. Blandin, J. Physique 41, 193 (1980). 